\title{
Artrodesis combinada anterior y posterior en paciente con parálisis cerebral atetósica que desarrolla mielopatía cervical degenerativa. Caso clínico y revisión de
} la literatura

\author{
G. Pancucci; P. Miranda-Lloret; M.E. Plaza-Ramírez; A. López-González; V. Rovira-Lillo y A. Beltrán-Giner \\ Unidad de patología raquimedular. Servicio de Neurocirugía. Hospital Universitario La Fe. Valencia.
}

\section{Resumen}

La mielopatía cervical espondiloartrósica es una complicación frecuente en pacientes jóvenes afectados por parálisis cerebral infantil (PCI) que presentan un importante componente distónico y atetósico. Su tratamiento quirúrgico es problemático, caracterizado por tasas elevadas de fracaso precoz de la artrodesis, tanto en los abordajes anteriores como en los posteriores a la columna. Ofrecemos una revisión histórica del tratamiento de la mielopatía cervical en este subgrupo de pacientes, aportando un caso clínico en el que se ha decidido descomprimir y realizar artrodesis tanto por la vía anterior como por la vía cervical posterior, con fijación con tornillos a masas laterales, complementando el tratamiento quirúrgico con la inyección de toxina botulínica en el seguimiento postoperatorio, logrando un buen resultado clínico.

PALABRAS CLAVE. Parálisis cerebral atetósica. Espondilosis cervical. Fusión espinal. Descompresión quirúrgica.

Combined Anterior-Posterior arthrodesis en patient with athetoid cerebral palsy who developed spondylotic cervical mielopathy. Case Report and review of literature

\section{Summary}

Spondylotic cervical mielopathy is a common complication in young patients with Cerebral Child Palsy with an important dystonic and athetoid component. Its surgical treatment is a challenge, due to elevate incidence of early faliure of the arthrodesis, both in anterior and posterior approaches. We report an historical review about the treatment of cervical mielopathy in this subgroup of patients and a clinical case in which we decided to realize decompression and arthtrodesis

Recibido: 28-01-10. Aceptado: 6-12-10 by a combined anterior and posterior approach, with lateral-mass screw placement, using botulinium toxin injections in the postoperative period, achieving a good clinical outcome.

KEY WORDS. Athetoid cerebral palsy. Cervical spondylosis. Spine fusion. Surgical decompression.

\section{Caso clínico}

Paciente de 39 años, mujer, afectada por parálisis cerebral infantil. La paciente presenta importante componente distónico y atetósico. No hay déficit cognitivo y la paciente es independiente para las actividades básicas de la vida diaria: vive sola, deambula sin ayuda, presenta un buen funcionamiento social (trabaja como administrativa).

La clínica empieza a principios de mayo 2009, con disestesias y pérdida de fuerza progresiva en las cuatro extremidades, asociado con dolor cervical de nivel 4 en la Dennis Pain Scale. Tras acudir en varias ocasiones a urgencias, en julio de 2009 es finalmente valorada por neurología, e ingresada para estudio. A la exploración destaca hipoestesia con distribución C2 izquierda, fuerza en extremidad superior izquierda de $3 / 5$ ( $1 / 5$ a nivel distal, con especial afectación de la extensión de muñeca), paraparesia $4 / 5$ en miembro inferior derecho y $3 / 5$ en el izquierdo. La paciente, que anteriormente deambulaba de forma independiente, se reduce a deambular con ayuda y, progresivamente, se queda en silla de ruedas o en la cama. No hay pérdida de control de esfínteres en ningún momento.

Se realiza un RM de columna cervical (Fig. 1, 2), que demuestra una estenosis grave de canal cervical, con una voluminosa hernia discal C3-C4 y alteración en la señal compatible con mielopatía a nivel C3-C5.

Valorado el caso por neurocirugía, se decide tratamiento quirúrgico. Los objetivos son lograr una descompresión de la médula cervical y garantizar una artrodesis estable a largo plazo. Para alcanzarlos, se plantea una cirugía en dos tiempos. En el primer tiempo (Fig. 3) se realiza un abordaje cervical anterior clásico, optando por una discectomía C3-C4 con osteofitectomía y fijando con prótesis de peek 

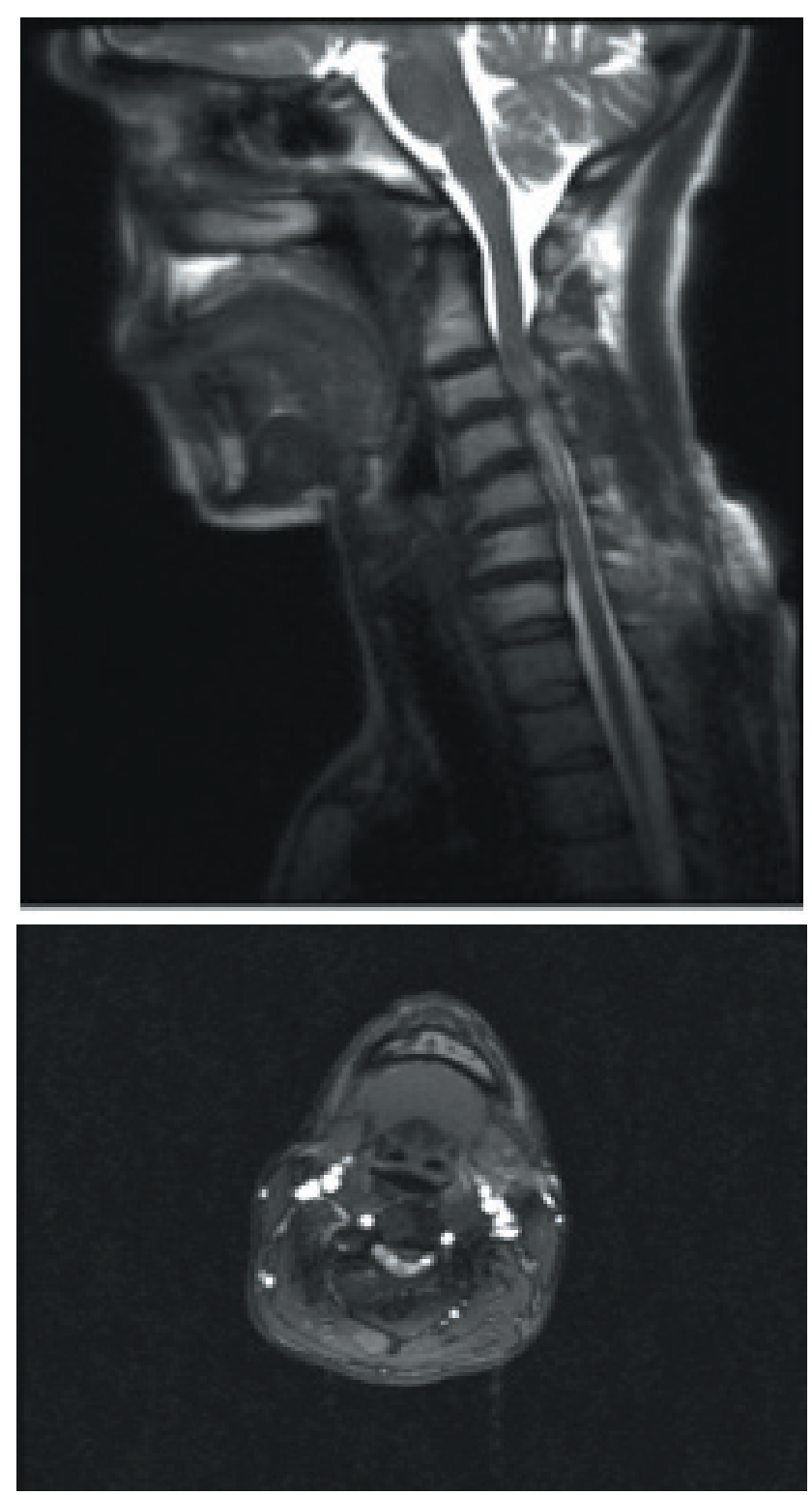

Figuras 1 y 2. RM preoperatoria, secuencias T2 sagital (1) y axial (2). Se aprecia un importante estrechamiento del canal medular cervical a nivel C3-C4, con componente anterior y posterior. Hiperintensidad intramedular C3 hasta C5 compatible con mielopatía.

(con hidroxiapatita cálcica trifosfato en su interior) y placa cervical anterior. En un segundo tiempo en el mismo procedimiento quirúrgico (Fig. 4) se realiza fijación con tornillos a masas laterales de C3 y C4 y laminectomía de C3 con flavectomía ampliada, sin complementar la artrodesis con hueso autólogo ni con sustituto óseo. Toda la cirugía se ha monitorizado con potenciales evocados motores y somatosensoriales, que en ningún momento han mostrado alteraciones en relación con los procedimientos. En los controles Rx y TAC (Fig. 5, 6) se aprecia el correcto posi-
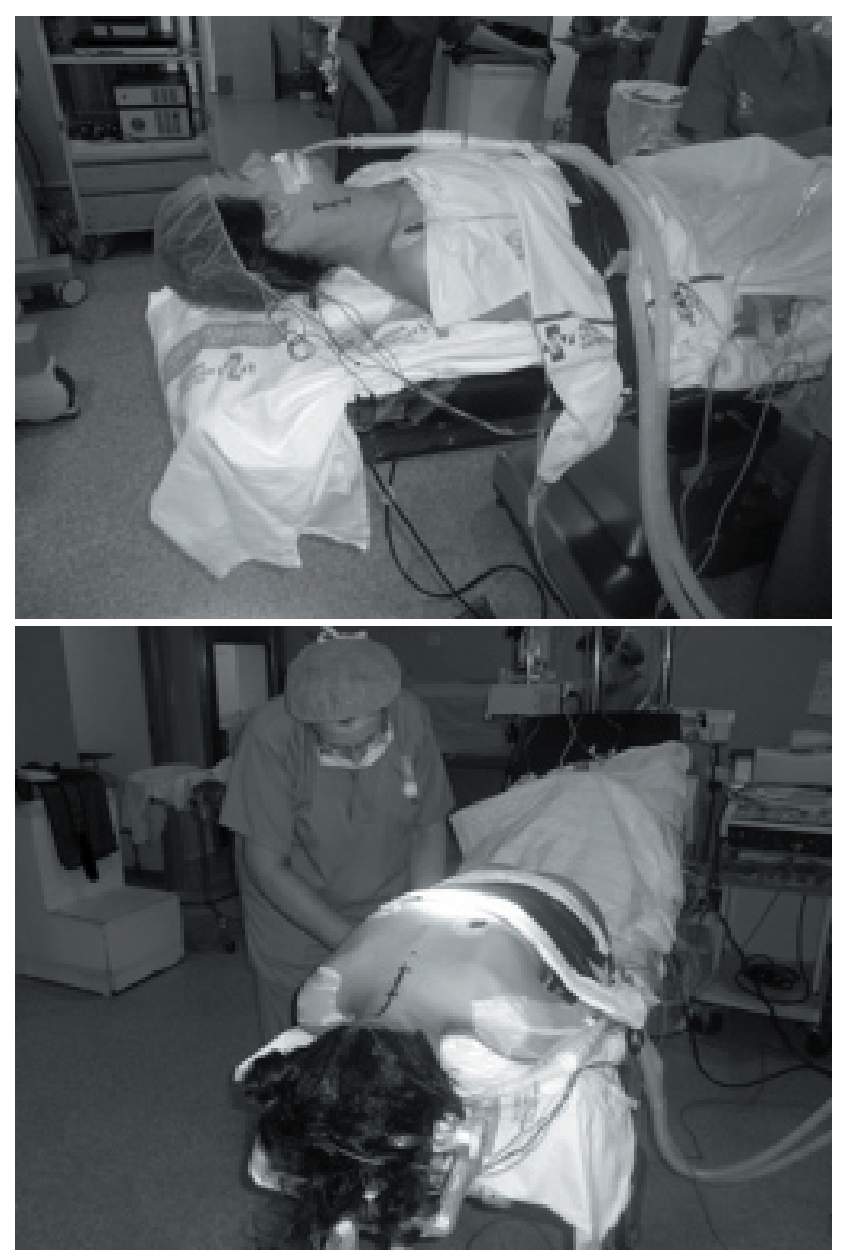

Figuras 3 y 4. Posición quirúrgica en el abordaje anterior (3), y posterior (4).

cionamiento del instrumental de fijación.

En el postoperatorio inmediato se han empleado infiltraciones de toxina botulínica para relajar la musculatura cervical (300 UI im bilateralmente en el borde medial del ECM). No ha sido necesario continuar este tratamiento tras el alta hospitalaria. La paciente comenzó rehabilitación inmediata en la unidad de lesionados medulares de nuestro centro, continuando, después del alta de forma ambulatoria.

A los tres meses el dolor cervical ha bajado en intensidad (de 4 a 2 en la Dennis Pain Scale), la puntuación motora es de 89/100 con lesión medular grado D en la clasificación ASIA. La paciente ha vuelto a deambular, con ayuda de andador, es autosuficiente para las ABVD y ha experimentado importante mejoría en la fuerza de la extremidad superior izquierda. En la RM de control a los 3 meses (Fig. 7) se aprecia la efectiva descompresión del canal cervical. En el último control, a los seis meses (Fig. 8), la paciente no se queja de dolor cervical, y su autonomía ha mejorado de forma llamativa, pudiendo reincorporarse a 


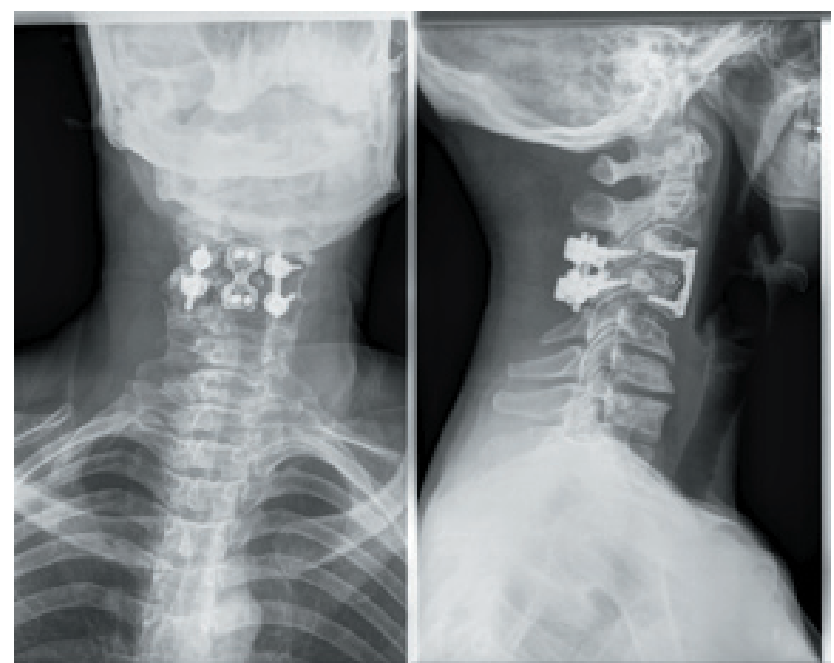

Figura 5. Control Rx postoperatorio.

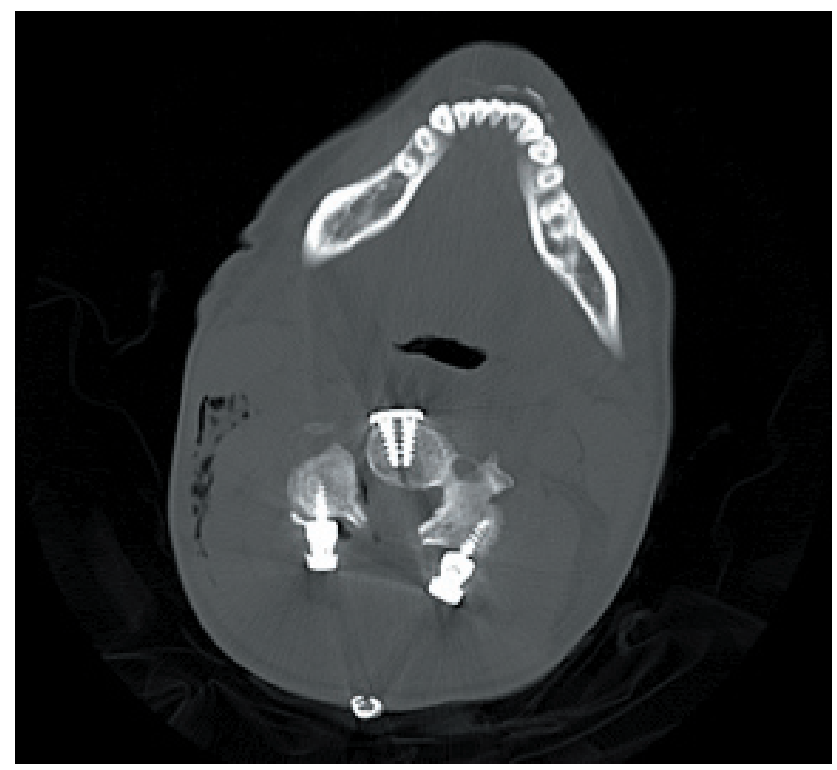

Figura 6. Control TC postoperatorio.

su puesto de trabajo.

\section{Discusión}

En los pacientes con parálisis cerebral infantil, hay una incidencia de espondiloartrosis cervical elevada y en edades precoces en comparación con la media de la población. Las posturas distónicas y los movimientos atetósicos producen fuerzas anormales de rotación y flexión que conllevan una inestabilidad dinámica en todos los niveles cervicales, que acaba generando cambios espondilóticos en edades juveniles. Los niveles más frecuentemente afectados en este tipo de paciente son C3-C4 y C4-C5 $5^{4,15,22,23}$. Esta característica es diferencial con respecto a la común enfermedad degenerativa cervical en pacientes añosos, que

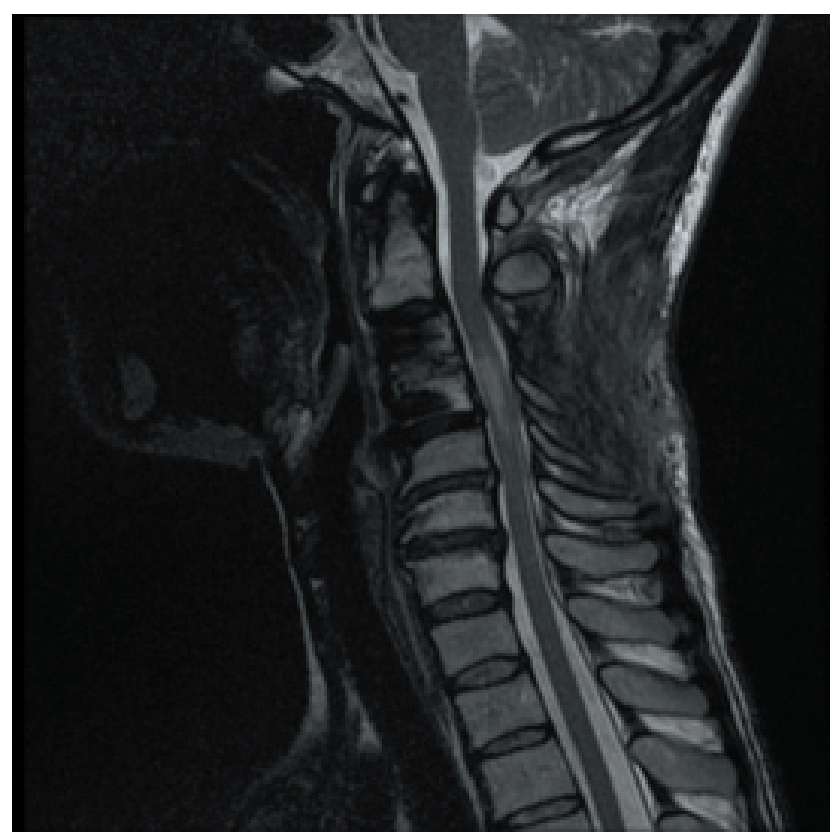

Figura 7. RM postoperatoria, a los 3 meses; secuencia T2 sagital. Desaparición de la estenosis a nivel de C3-C4; persiste la alteración de señal compatible con mielopatía.

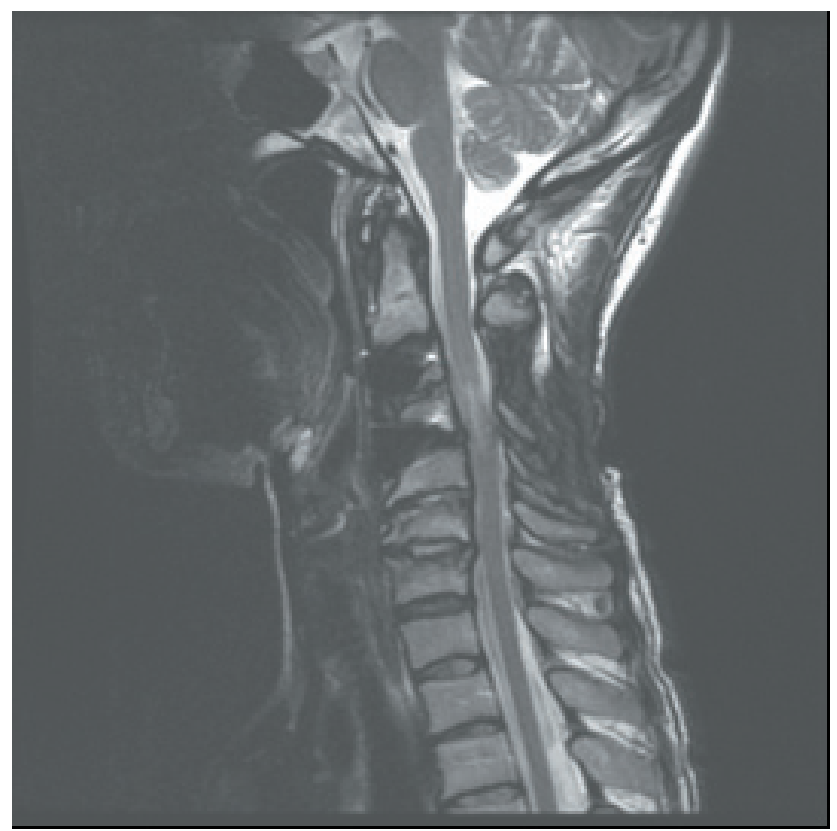

Figura 8. RM a los 6 meses; se aprecia la liberación del canal y quizá leve disminución de la señal mielopática.

afecta con más frecuencia a segmentos más distales (C6 y C7 sobre todo). Según Harada $\mathrm{T}^{7}$ en este subgrupo de pacientes, comparado con controles sanos, hay una incidencia de degeneración discal de 8:1, y de inestabilidad de los segmentos C3-C4 y C4-C5, de 6:1.

Si bien la cervicoartrosis en este tipo de pacientes es precoz, y por lo tanto sus síntomas se produzcan en edades 
más tempranas ${ }^{3,15}$, debido a la patología de base, muchas veces los síntomas de la mielopatía y de la radiculopatía pasan desapercibidos, y, por lo tanto, el diagnóstico es tardío. En el caso clínico descrito, por ejemplo, la paciente afectada por PCI lleva una vida autónoma (vive sola en un piso) e incluso tiene un papel activo en el mundo laboral. Los síntomas de la mielopatía reducen su actividad y aumentan su dependencia hasta limitarla en una silla de ruedas y, finalmente, en una cama. La progresión clínica es de tipo subagudo, pero el diagnóstico se realiza sólo después de tres meses desde que la paciente empezó a notar los primeros síntomas.

El primer caso comunicado de mielopatía cervical en pacientes con PCI fue publicado por Anderson en $1962^{2}$. Inicialmente los resultados del tratamiento quirúrgico han sido pobres, y por lo tanto se han intentado tratamientos conservadores como medicación miorrelajante y ortesis cervicales. Las medidas conservadoras han demostrado su ineficacia e incluso pueden ser peligrosas, debido a la imposibilidad para estos pacientes de mantener una inmovilización cervical ${ }^{2,15}$. Los primeros intentos quirúrgicos han sido abordajes posteriores, casi exclusivamente laminectomías descompresivas ${ }^{2,14}$. Esta técnica produce una mejoría inicial, pero a corto-mediano plazo se asocia con un aumento de la inestabilidad y fracaso precoz con consecuente recidiva de los síntomas; de hecho varios autores consideran contraindicada la laminectomía en este subgrupo de pacientes ${ }^{2,11,14,20,22}$. De forma aislada, la laminoplastia ha demostrado eficacia, asociada a la relajación muscu$\operatorname{lar}^{20}$. Posteriormente se comenzaron a utilizar técnicas de descompresión y fusión anterior y/o posterior, con resultados controvertidos, pero no desalentadores ${ }^{5,6,8,12,15,16}$. La descompresión y fusión por vía exclusivamente anterior ha demostrado un buen resultado clínico ${ }^{17}$; por otro lado en varias series, que han llevado un seguimiento a largo plazo, se ha demostrado fracaso de la artrodesis y reincidencia de los síntomas en un número importante de pacientes. Además se han reportado casos de deterioro (cifosis) de los niveles adyacentes e inestabilidad atlo-axial ${ }^{12,17}$. Como otra complicación, la artrodesis anterior de estos pacientes, sobre todo si es de más de un nivel, se asocia con tasas más elevadas de fracturas vertebrales y pseudoartrosis ${ }^{10}$.

En la actualidad se reconoce que en la cirugía de la mielopatía espondiloartrósica, a parte de la descompresión del neuroeje, garantizar una artrodesis duradera es problemático. De hecho, las alteraciones en el tono y la movilidad de la musculatura cervical generan un estrés que es responsable del fallo precoz de la artrodesis. Por esto varios autores abogan por la utilización de sistemas de fijación rígida externa en el postoperatorio y durante periodos más o menos $\operatorname{largos}^{15,22}$. Otros autores han intentado utilizar relajantes musculares en el perioperatorio, como la toxina botulínica ${ }^{1}$, para reducir el hipertono y los movimientos atetósicos, que en el postoperatorio suelen tener incluso un aumento en intensidad debido al dolor postquirúrgico. Recientemente, con el objetivo de reducir las tasas de fracaso precoz de la instrumentación, se han realizado artrodesis anteriores y posteriores, que garantizan más estabilidad y no necesitan sistemas de inmovilización rígida (como el halo vest) en el postoperatorio ${ }^{5,12}$.

En cuanto a la técnica quirúrgica llevada a cabo en el caso comentado, cabe destacar la hipertrofia músculoligamentosa y ósea, tan importante que ha obligado a trabajar con motor neumático: la probable explicación es que el estrés crónico al que han estado expuestos los tejidos blandos y óseos de la región cervical, debido a los movimientos involuntarios, haya generado una hipertrofia patológica de los tejidos implicados. La intervención se ha realizado en dos distintos tiempos quirúrgicos, pero en la misma sesión: una vez terminado el abordaje anterior, sin despertar a la paciente, se ha preparado el campo quirúrgico para el abordaje posterior. La duración de la intervención ha sido de 6 horas y media. La descompresión anterior del espacio C3$\mathrm{C} 4$ se ha realizado con técnica microquirúrgica, utilizando motor neumático para la osteofitectomía. La artrodesis con prótesis de peek y placa anterior no ha tenido incidencias. En cuanto al abordaje posterior (artrodesis y laminectomía con flavectomía ampliada C3-C4), cabe destacar que se ha preferido utilizar tornillos a masas laterales y barras con respecto a los alambres; de hecho los tornillos garantizan una estabilidad interna inmediata, obviando la necesidad de sistemas de inmovilización rígida externa ${ }^{21}$. La técnica utilizada para posicionar los tornillos ha sido la fijación a masas laterales según Magerl $^{9}$, excepto a nivel de C3 izquierdo, donde por pérdida de presa con la trayectoria de Magerl se ha utilizado el método de Roy-Camille ${ }^{19}$ como rescate, al tener un punto de entrada más medial y una trayectoria menos angulada.

Onari et al. ${ }^{15}$, en 2002, publican una de las series más largas de pacientes con espondiloartrosis cervical y PCI tratados con doble abordaje anterior y posterior, concluyendo que esta técnica mejora la función neurológica, incluso en pacientes con importantes movimientos involuntarios, sin necesitar fijación rígida externa. Por lo tanto en el caso clínico comentado, en el postoperatorio no se han utilizado casi sistemas de inmovilización rígida (la paciente ha llevado un collarín tipo Philadelphia, pero solo durante los 3 primeros días); en cambio se ha decidido practicar infiltraciones con toxina botulínica en el vientre anterior del músculo esternocleidomastoideo. Cabe destacar al respecto que varios autores han logrado buenos resultados en el control del dolor y de los movimientos involuntarios en el postoperatorio con la aplicación tópica de toxina botulínica $^{1,13,18}$, pero su eficacia en favorecer la consolidación de la artrodesis y en prevenir el deterioro tardío queda en un tema a discutir, ya que la duración del efecto farmacológico 
es breve. En nuestra experiencia, hemos apreciado una importante mejoría del cuadro álgico y una reducción de los movimientos espontáneos tras la administración de la toxina.

\section{Conclusiones}

El primer paso hacia el correcto tratamiento de los pacientes con espondilosis cervical en el contexto de una parálisis cerebral infantil es el enfoque diagnóstico precoz, ya que muchas veces la mielo-radiculopatía conlleva un empeoramiento clínico que puede pasar desapercibido debido a la enfermedad neurológica de base. La descompresión quirúrgica y la estabilización en este tipo de paciente es un reto para la cirugía. La fijación combinada antero-posterior parece acelerar la fusión y garantizar estabilidad en la región intervenida, reduciendo la tasa de fracaso de la artrodesis, en comparación con los abordajes exclusivamente anteriores o posteriores. Además, al no necesitar inmovilización externa rígida postoperatoria, reduce las molestias para el paciente y las complicaciones en relación con sistemas como el halo-chaleco. La miorrelajación con toxina botulínica en el perioperatorio disminuye el dolor y los movimientos involuntarios, y podría facilitar la fusión precoz. De todas maneras, son necesarias series más grandes y un seguimiento más largo, ya que, debido a la incidencia precoz de mielopatía, estos pacientes son operados en edades más jóvenes con respecto a la media, y por lo tanto, tienen un riesgo potencial de degeneración cervical tardía o de deterioro neurológico, porque a pesar de la estabilización lograda con la cirugía, persisten los movimientos involuntarios que favorecen la degeneración de la columna cervical.

\section{Bibliografía}

1. Adler, C.H., Zimmerman, R.S., Lyons, M.K., et al.: Perioperative use of botulinum toxin for movement disorder induced cervical spine disease. Mov Disord. 1996; 11: 79-81.

2. Anderson, W.W., Wise, B.L., Itabashi, H.H., Jones, M.: Cervical spondylosis in patients with athetosis. Neurology 1962; 12: 410-412.

3. Azuma, S., Seichi, A., Ohnishi, I., Kawaguchi, H., Kitagawa, T., Nakamura, K.: Long-Term Results of operative treatment for cervical spondylotic myelopathy in patients with athetoid cerebral palsy. An Over 10 year follow-up study. Spine 2002; 27: 943-948; discussion 948.

4. Cho, Y.J., Lee, M.H., Chang, S.K.: Cervical Radiographic Study in Adolescence Cerebral Palsy. J Korean Acad Rehabil Med 1998; 22: 543- 551.

5. Epstein, N.E.: circumferencial cervical surgery for spondylostenosis with kyphosis in two patients with athetoid cerebral palsy. Surg Neurol 1999; 52: 339-344.
6. Fuji, T., Yonenobu, K., Fujiwara, K., et al.: Cervical radiculopathy or myelopathy secondary to athetoid cerebral palsy. J Bone Joint Surgery Am. 1987; 69: 815-821.

7. Harada, T., Ebara, S., Anwar, M.N., Okawa, A., Kajiura, I., Hiroshima, K., Ono, K.: The cervical spine in athetoid cerebral palsy. A radiological study of 180 patients. J Bone Joint Surg Br. 1996; 78: 613-619.

8. Hirose, G., Kadoya, S.: Cervical spondylotic radiculomyelopathy in patients with athetoid-dystonic cerebral palsy. Clinical evaluation and surgical treatment. J Neurol Neurosurg Psychiatry 1984; 47: 775-780.

9. Jeanneret, B., Magerl, F., Ward, E.H., et al.: Posterior stabilization of the cervical spine with hook plates. Spine 1991; 16 (Suppl): S56-S63.

10. Kim, J.G., Kim, S.W., Lee, S.M., Shin, H.: Surgical results of the combined anterior and posterior approach in treatment of cervical spondylotic myelopathy. J Koren Neurosurg Soc 2006; 39: 188-191.

11. Ko, H.Y., Park-Ko, I.: Spinal cord injury secondary to cervical disc herniation in ambulatory patients with cerebral palsy. Spinal Cord 1998; 36: 288-292.

12. Lee, Y.J., Chung, D.S., Kim, J.T., Bong, H.J., Han, Y,M., Park, Y.S.: Surgical treatments for cervical spondylotic myelopathy associated with athetoid cerebral palsy. J Korean Neurosurg Soc. 2008; 43: 294-299.

13. Murozumi, M., Kurose, Y., Katayama, S., et al.: Muscle release of cervical flexor and extensor muscle in adult CP. Cent Jpn J Ortop Traumatol 1997; 40: 607-608.

14. Nishihara, N., Tanabe, G., Nakahara, S., et al.: Surgical treatment of cervical spondylotic myelopathy complicating athetoid cerebral palsy. J Bone Joint Surg Br. 1984; 66: 504-508.

15. Onari, K., Kondo, S., Mihara, H., Iwamura, Y.: Combined anterior and posterior fusion for cervical spondylotic myelopathy in patients with athetoid cerebral palsy. J Neurosurg 202; (1 Suppl) 97: 13-19.

16. Polk, J.L., Maragos, V.A., Nicholas, J.: Cervical spondylotic myeloradiculopathy in dystonia. Arch Phys Med Rehabil. 1992; 73: 389-392.

17. Pollak, L., Schiffer, J., Klein, C., Mirovsky, Y., Copeliovich, L., Rabey, J.M.: Neurosurgical intervention for cervical disk disease in dystonic cerebral palsy. Mov Disord 1998; 13: 713-717.

18. Racette, B.A., Lauryssen, C., Perlmutter, J.S.: Preoperative treatment with botulinum toxin to facilitate cervical fusion in dystonic cerebral palsy. Report of two cases. J Neurosurg 1998; 88: 328-330.

19. Roy-Camille, R., Saillant, G., Lavile, C., Benazet, J.P.: Treatment of lower cervical spine injuries C3 to C7. Spine 1992; 17 (Suppl): S442-446.

20. Ueda, Y., Yoshikawa, T., Koizumi, M., Iida, J., Miyazaki, K., Nishiyama, S., et al.: Cervical Laminoplasty Combined with muscle release in patients with athetoid cerebral palsy. Spine 2005; 30: 2420-2423. 
21.Wellman, B.J., Follet, K.A., Traynelis, V.C.: Complications of posterior articular mass plate fixation of the subaxial cervical spine in 43 consecutive patients. Spine 1998; 15; 23: 193-200.

22. Wong, A.S., Massicotte, E.M., Fehlings, M.G.: Surgical treatment of cervical myeloradiculopathy associated with movement disorders: indications, technique, and clinical outcome. J spinal Disord Tech 2005; 18: S107-S114.

23. Yong-Jeon, L., Dong-Sup, C., Jong-Tae, K., Ho-Jin, B., Young-Min, H., Young-Sup, P.: Spondylotic Myelopathy associated with Athetoid Cerebral Palsy.
Pancucci, G.; Miranda-Lloret, P.; Plaza-Ramírez, M.E.; López-González, A.; Rovira-Lillo, V.; Beltrán-Giner, A.: Artrodesis combinada anterior y posterior en paciente con parálisis cerebral atetósica que desarrolla mielopatía cervical degenerativa. Caso clínico y revisión de la literatura. Neurocirugía 2011; 22: 245-250.

Correspondencia: Unidad de patología raquimedular. Servicio de Neurocirugía. Hospital Universitario La Fe. Avenida Campanar, 21. 46009 Valencia, Comunitat Valenciana.

E-mail: panc82@hotmail.com 